\title{
Die geographischen Grenzen abstrakter Gleichheit
}

\author{
Bernd Belina \\ Institut für Humangeographie, Goethe-Universität Frankfurt, Frankfurt am Main, Germany \\ Correspondence: Bernd Belina (belina@em.uni-frankfurt.de)
}

Received: 18 January 2019 - Revised: 6 October 2020 - Accepted: 9 October 2020 - Published: 10 November 2020

\begin{abstract}
Equality, a concept so central to democratic societies, is being scrutinized in a critical manner in this paper. It argues that the spatial borders of territorial states are also the limits of the validity of the principle of abstract equality, of its ideological productivity as well as its emancipatory potential. The paper discusses the Marxist critique of the limitations of the merely abstract, formal understanding of equality that is inscribed into the structures of democratic states, and the ways in which both the Marxist tradition and current theories of radical democracy find an emancipatory potential in the demand for abstract equality that makes possible going beyond its very abstractions. The focus of these discussions is on how spatiality is integrated into theories of radical democracy on the level of theory. The paper suggests that combining the insights on the productivity of spatial forms from discussions in human geography with the critique of merely abstract equality is a decidedly geographical contribution to the development of theories of the political.
\end{abstract}

\section{Introduction}

Die Struktur des Wirtschaftssystems wirkt sich auf alle menschlichen Beziehungen und selbst die innerste Verfaßtheit des Individuums aus. Insofern spiegelt der alles durchdringende Zug der gesellschaftlichen ,Entfremdung ' weitgehend das Wesen einer Warenwirtschaft wider, in der der Mensch als Produzent und Konsument von Waren und nicht als Subjekt seiner Gesellschaft erscheint (Adorno, 2019:45).

Gleichheit gehört zu den Grundlagen demokratischer Gesellschaften. Ziel dieses Beitrages ist es, diesen schillernden Begriff kritisch zu hinterfragen und $\mathrm{zu}$ argumentieren, dass die räumliche Organisation kapitalistischer Gesellschaften von entscheidender Bedeutung für die Grenzen seiner Geltung ebenso wie seines emanzipatorischen Potentials ist. Anlass dieser Diskussion ist die weit geteilte Einschätzung, dass die in Deutschland jüngst politisch so erfolgreiche Partei „Alternative für Deutschland“ (AfD) das „,universelle Gleichheitspostulat der Aufklärung [...] infrage stellt" (Salzborn, 2017:166). Anderswo habe ich argumentiert, dass dem nicht generell so ist, sondern dass der breite Zuspruch zu AfD-Positionen gerade auf einer selektiven Infragestellung abstrakter Gleichheit basiert, die an deren Grundlagen in den fundamentalen Strukturen kapitalistischer Vergesellschaftung gerade nicht rüttelt (Belina, 2020a). Zentral ist die Ungleichheit zwischen „Inländern“ und „Ausländern“, die in Deutschland infolge des ,langen Sommers der Migration“ 2015 - erneut - im Zentrum politischer Debatten steht. So bezeichnet der Bundesinnenminister Migration als „Mutter aller Probleme“ und äußert „Verständnis“ (SPON, 2018) für die „,rechtsautoritären Massenmobilisierungen“ (Intelmann, 2019:189) in Chemnitz 2018. Dies illustriert, dass auch die selbsterklärte Mitte politische Fremdenfeindlichkeit und Rassismus bedienen will, von denen aus empirischen Studien bekannt ist, dass sie bis weit in die Mitte der Gesellschaft geteilt werden (Zick et al., 2019:66-73). Die neoliberale Gleichheit „der Isolation und der Entrepreneurisierung verantwortlicher Einheiten und Individuen“ (Brown, 2018:152) hingegen wird in AfDPositionen radikalisiert (vgl. Dietl, 2017).

Die Betonung dieser selektiven Infragestellung abstrakter Gleichheit durch die AfD interveniert in die Debatte um die Frage, ob „ökonomische“ oder „kulturelle“ Erklärungen den Aufstieg der AfD besser erklären können (Belina, 2020b). Jenseits solcher Vereinfachungen folgt dieser Beitrag Adornos eingangs zitierter Position, entnommen seinen Erläuterungen zur klassischen Studie The Authoritarian Personality von 1950, deren Interesse dem ,poten- 
tiell faschistische[n] Individuum“ (Adorno et al., 2017:1) ${ }^{1}$ galt, „dessen Struktur es besonders empfänglich für antidemokratische Propaganda macht" (ebd.). Im Folgenden steht die andere Seite des Verhältnisses IndividuumWirtschaftssystem im Zentrum, genauer: dass und wie die durch das Wirtschaftssystem produzierte abstrakte Gleichheit der Individuen einerseits dessen Widersprüche prozessierbar macht, andererseits eine objektive Grenze in den räumlichen Grenzen der territorialen Form ihre Organisation findet - und wie diese Widersprüchlichkeit dazu führt, dass Migrationsabwehr, Fremdenfeindlichkeit und Rassismus plausibel wirken und hegemonial werden können.

Theoretischer Ausgangspunkt der folgenden Überlegungen ist die materialistische Analyse, nach der die grundlegende Art und Weise, in der kapitalistische Gesellschaften organisiert sind, mithin ihre sozialen Formen, ihre Widersprüche zugleich verschleiern und ,prozessierbar“ (Hirsch, 1994:161) machen (vgl. Belina, 2019). Diese Leistung der sozialen Formen erklärt, warum die in den Jahrzehnten der Neoliberalisierung verschärften ökonomischen Widersprüche und Ungleichheiten zwar ein generelles ,soziales Angstgefühl“" (Hall et al., 1978) hervorgebracht haben, das durch die globale Finanz- und Wirtschaftskrise ab 2007 noch einmal verstärkt wurde, dass es aber erst die sichtbare praktische Infragestellung der räumlichen Grenze der „Nationform“ (Balibar, 1990, 2003) im Jahr 2015 durch die „Autonomie der Migration“ (Georgi, 2019) war, die die migrations- und migrant*innenfeindliche AfD-Propaganda auf fruchtbaren Boden fallen ließ. Weil die sozialen Formen in der Raumform des mit Bedeutung aufgeladenen Territoriums organisiert sind, also im Nationalstaat, finden die Leistungen der sozialen Formen und die abstrakte Gleichheit der Markt-, Rechts- und staatsbürgerlichen Subjekte ihre Grenze an der räumlichen Grenze des Staates (Belina, 2020a).

Ausgehend von der Erklärung der Menschen- und Bürgerrechte von 1789 (Kapitel 1) wird im Folgenden zunächst die Kritik an der nur abstrakten Gleichheit bei Marx, Adorno und Brown entwickelt (Kapitel 2). Deren Aktualität dient als Grundlage der kritischen Auseinandersetzung mit den Beiträgen von Laclau/Mouffe, Rancière und Balibar, die in unterschiedlicher Weise in der Ausweitung abstrakter Gleichheit über sich selbst hinaus den Kern von radikaler Demokratie sehen (Kapitel 3). Im Fazit wird mit Verweis auf Adorno und Adamczak angedeutet, was aus der kritischen Diskussion folgt (Kapitel 4).

Ausgehend von der Beobachtung Harveys (1999:xxii), dass Fragen des Raums ,in die Marx'sche Theorie in weit expliziterer Weise hineingeschrieben werden [müssen], als Marx selbst das geleistet hat", besteht der geographische Beitrag des Folgenden darin, die wesentliche Räumlichkeit der sozialen Formen des Kapitalismus auf Ebene der The-

\footnotetext{
${ }^{1}$ Alle Hervorhebungen in Zitaten wie im Original, alle Übersetzungen durch den Autor.
}

orie in Diskussionen um Möglichkeiten emanzipatorischer Politik stark zu machen.

\section{9}

Gleichheit, wie wir sie heute verstehen, kommt mit den bürgerlichen Revolutionen des 18. und 19. Jahrhunderts als Gleichheit der Bürger*innen vor dem Recht in die Welt. Als ihr Gründungsdokument wird die Erklärung der Menschenund Bürgerrechte von 1789 angesehen. Über diese schreibt der Historiker George Lefebvre (1989:195):
[Die] Revolution bringt aber [neben der Freiheit] auch die Gleichheit vor dem Gesetz; ohne sie wäre die Freiheit ja nur ein weiteres Privileg der Mächti- gen. Für die Franzosen von 1789 sind Freiheit und Gleichheit untrennbar verbunden, im Grunde Wörter, die dasselbe bedeuten. Hätten sie wählen müssen, so wäre ihnen die Gleichheit am wichtig- sten gewesen, und wenn die Bauern, die ja ihre überwältigende Mehrheit bildeten, der Freiheit zu- jubelten, so dachten sie dabei an die Abschaf- fung der Macht des Grundherrn, der jetzt einfacher Bürger sein sollte, also im Grunde an die Gleich- heit.

Lefebvre (1989) betont den Zusammenhang, ja die tendenzielle Identität von Freiheit und Gleichheit als Forderungen des Dritten Standes, die das Bürgertum mit den Bauern gegen Aristokratie und Klerus teilt - und auch, dass diese „keinen Augenblick angenommen [haben], die Menschenund Bürgerrechte könnten lediglich für die Franzosen gelten. [...] Freiheit und Gleichheit seien das gemeinsame Gut der Menschheit" (ebd.:195). Allerdings zeigte sich der Widerspruch zwischen den im territorialen Nationalstaat geltenden Bürgerrechten und den universellen Menschenrechten schon bald in concreto: Als 1830/31 über 10000 Pol*innen nach Frankreich flohen, wo die Nationalversammlung 1793 verkündet hatte, dass ,,das französische Volk“ allen um ihrer Freiheit willen aus ihrem Vaterland vertriebenen Ausländern Asyl gewährt“ (Noiriel, 1994:14), war es für die Parlamentsmehrheit „undenkbar, Ausländern die gleichen Rechte wie Franzosen zuzugestehen“ (ebd.:23).

Als marxistisch orientierter Historiker zeigt Lefebvre (1989:199) außerdem, wie sich die gemeinsame revolutionäre Position im turbulenten Jahr 1789 in Kämpfen des proletarischen Volkes auf der Straße und der bürgerlichen Vertreter*innen in der Nationalversammlung erst konstituieren muss:

Das Volk hat zwar durch sein Eingreifen die Versammlung gerettet, aber es wäre ein Irrtum $\mathrm{zu}$ meinen, es habe die gleichen Vorstellungen gehabt wie das Bürgertum. Es hatte eigene Motive. Eines war die Abschaffung des Feudalsystems, aber es wollte zugleich die alte, stark regle- 
mentierte Wirtschaftsordnung wieder einführen, die einer Entwicklung des Kapitalismus im Wege stand und von Regierung, Großgrundbesitz und Großbürgertum im Laufe des 18. Jahrhunderts nach und nach abgebaut worden war. [...] Ganz allgemein machte die Erklärung der Gleichheit der Rechte erst die Ungleichheit der Möglichkeiten deutlich, und da diese zum Teil auf der Ungleichheit des Vermögens beruhten, konnten erste Anzeichen eines zugleich politischen und sozialen Konflikts zwischen Besitzenden und Proletariern nicht ausbleiben.

An eben diesem Widerspruch zwischen denen, die gemeinsam die Revolutionen machten, setzt die marxistische Kritik der Gleichheit als nur abstrakte, formale, die realen Ungleichheiten verschleiernde an.

\section{Kritik der abstrakten Gleichheit}

Gleichheit ist „kein ,marxistischer“ Begriff“ (Mainfroy, 2001:846). Im Gegenteil wird in der marxistischen Tradition jede Norm basierend auf bloß abstrakte Gleichheit als die realen Ungleichheiten verschleiernd kritisiert. Gleichwohl ringen Theoretiker*innen in dieser Tradition mit der Frage, in welcher Weise die Vorstellung von Gleichheit emanzipatorisch produktiv ist und wie eine reale Gleichheit aussehen könnte.

Bereits in Zur Judenfrage, geschrieben 1843, kritisiert Marx (1970:362) „die sogenannten Menschenrechte“ in ,ihrer authentischen Gestalt, [...] welche sie bei ihren Entdeckern, den Nordamerikanern und Franzosen, besitzen“ (ebd.), dahingehend, dass sie „nichts anderes sind als die Rechte des Mitglieds der bürgerlichen Gesellschaft, d. h. des egoistischen Menschen, des vom Menschen und vom Gemeinwesen getrennten Menschen“ (ebd.:364). Obschon dem Namen nach für alle Menschen geltend, sind sie de facto vom Staat garantierte Rechte seiner Bürger*innen auf Verfolgung ihrer individuellen Privatinteressen, allem voran dem nach der Mehrung ihres kapitalistischen Reichtums: „Die praktische Nutzanwendung des Menschenrechtes der Freiheit ist das Menschenrecht des Privateigentums" (ebd.), und Gleichheit ,ist nichts als die Gleichheit der [...] liberté, nämlich: daß jeder Mensch gleichmäßig als solche auf sich ruhende Monade betrachtet wird“ (ebd.:365). Die Menschenrechte sehen vom ,wahre[n] Mensch[en]“ ab und reduzieren ihn auf die „Gestalt des egoistischen Individuums, [...] des abstrakten citoyen" (ebd.:370). Aufgrund dieser „Abstraktion“ (ebd.) sind die staatlich zuerkannten Rechte ,[w]eit entfernt, diese faktischen Unterschiede [der Geburt, des Standes, der Bildung, der Beschäftigung] aufzuheben" (ebd.:354). Mit dieser vernichtenden Kritik der Menschenrechte und insbesondere der Gleichheit, die Menschen als Marktindividuen isoliert, zielt Marx explizit gegen die Hoffnung, dass mit ihnen „die menschliche Emanzipation“ (ebd.:370) befördert würde.

Auch für den späten Marx (1969a:21) der Kritik des Gothaer Programms von 1875 ist ,alles Recht“ aufgrund der abstrakten Gleichheit, die es unterstellt, ,ein Recht der Ungleichheit".

Das Recht kann seiner Natur nach nur in Anwendung von gleichem Maßstab bestehn; aber die ungleichen Individuen (und sie wären nicht verschiedne Individuen, wenn sie nicht ungleiche wären) sind nur an gleichem Maßstab meßbar, soweit man sie unter einen gleichen Gesichtspunkt bringt, sie nur von einer bestimmten Seite faßt. (ebd.).

Indem man dies tut, ,ist jede allgemeine Regel, die solche Unterschiede zwischen Individuen, die eine unterschiedliche Behandlung begründen könnten, unberücksichtigt lässt, allein aus diesem Grund ,abstrakt ‘ und ,einseitig'(Lukes, 1997:30).

Diese Kritik bleibt in der marxistischen Tradition zentral. Abstrakte Gleichheit konstituiert, so Adorno (2018:445), die „fortwährende Ungerechtigkeit durchs Recht“. Sie ist „Ideologie [...], weil sie die ungemilderten Unterschiede gesellschaftlicher Macht, die von Hunger und Überfluß, von Geist und fügsamem Schwachsinn an den Menschen unterschlägt" (ebd.:457). Diese Kritik ist und bleibt aktuell, wie etwa Brown (2018:246f.) prominent ausführt:

[Die] liberale Demokratie [...] hat [...] durch ihre politischen und rechtlichen Abstraktionen die Macht und die Privilegien der gesellschaftlich dominierenden Kräfte gesichert [...]. Durch ihren formalen Kontext und die Inhaltsneutralität erscheinen liberal-demokratische Ideale des Personseins, der Freiheit und Gleichheit universell, obwohl sie mit Normen des bürgerlichen, weißen, männlichen, heterosexuellen Paternalismus gesättigt sind.

Brown und zahlreiche andere Vertreter*innen aktueller Debatten der politischen Philosophie stehen in dieser Hinsicht in der marxistischen Tradition und gehen im Bemühen, diese zu aktualisieren, über sie hinaus. Oft nur implizit ist damit die Analyse der sozialen Formen des Kapitalismus, die auf den praktisch wahr gemachten Abstraktionen von Freiheit und Gleichheit basieren, Ausgangspunkt der Überlegungen.

Den Kern dieser sozialen Formen, also der Art und Weise, in der Gesellschaft im Kapitalismus organisiert ist, formuliert Adorno (2003:57) folgendermaßen:

Das, was Gesellschaft eigentlich zu einem Gesellschaftlichen macht, wodurch sie im spezifischen Sinn sowohl begrifflich [...] wie auch real konstituiert wird, das ist das Tauschverhältnis, das 
virtuell alle Menschen, die an diesem Begriff von Gesellschaft teilhaben, zusammenschließt.

Alle, die am die Gesellschaft konstituierenden kapitalistischen Tausch teilnehmen, werden auf diese Weise abstrakt gleich: „Als Subjekte des Austauschs ist ihre Beziehung daher die der Gleichheit" (Marx, 1983:166f.). Jeder Mensch, der in dieser Form ökonomisch agiert, wird zum „Tauschsubjekt“ (Blanke et al., 1974:71), das mit jedem Tausch ,willensmäßige Beziehungen voneinander unabhängiger, einander gleicher Einheiten, juristischer Subjekte“ (Paschukanis, 1929:96) eingeht. Auf dieser Basis schließlich werden die Subjekte zu „StaatsbürgerInnen“ (Hirsch, 1994:169), und nur als solche sind sie abstrakt gleich, obschon sie aufgrund ihrer Klassenlage und anderer struktureller Positionierungen real ungleich sind. Als Tausch-, Rechts- und staatsbürgerliche Subjekte sind sie - das war der Ausgangspunkt von Marx in Zur Judenfrage - zudem vereinzelte, worauf neoliberale Ideologie und Praxis aufbauen und was sie radikalisieren, um reale ökonomische Ungleichheit zu legitimieren (Brown, 2018).

Die real hergestellten Unterschiede zwischen diesen vereinzelten Subjekten nicht zu unterschlagen, sondern im Gegenteil zu überwinden, ist bei Marx und bleibt bis heute Ziel der Kritik. Erst ,[i]n einer höheren Phase der kommunistischen Gesellschaft“, so Marx (1969a:21), „kann der enge bürgerliche Rechtshorizont ganz überschritten werden und die Gesellschaft auf ihre Fahne schreiben: Jeder nach seinen Fähigkeiten, jedem nach seinen Bedürfnissen!“ In dieser Weise die Individualität der Bedürfnisse im Kontext der Unterschiede der Lebensverhältnisse ins Zentrum zu stellen und zum Maßstab für eine andere Gesellschaft zu machen, ist nach Lukes (1997:30) Ausdruck einer ,romantischen Gesamtsicht". Diese Kritik verweist auf die Frage, wer denn der o. g. ,wahre Mensch“ (Marx, 1970:370) ist, von dem die Gleichheit abstrahiert. Bei Marx unterscheidet er sich fundamental von der Verabsolutierung des Individuellen in der romantischen Gegnerschaft zur Aufklärung, die, so Mishra (2017:78), gegen die „neue materialistische, individualistische und imperialistische Zivilisation im Namen lokaler religiöser und kultureller Wahrheit und spiritueller Tugend“ den „Wert des Einsseins - mit sich selbst, den anderen und der Natur" (ebd.:211) hochhielt - Positionen, die Mishra zu Folge bis heute eine unheilvolle Verbindung aus Ressentiment, Antielitismus, Männlichkeitswahn, Nation und Gewalt stiften, wie ihn auch die AfD vertritt. Entgegen Mishras (2017:192) Unterstellung, dass auch die „marxistische Dialektik ihren Ursprung im Ressentiment“ habe und Marx einer romantisierenden Vorstellung des Individuums anhing, ist festzuhalten, dass bereits der junge Marx (1969b:6) - also jener, dem romantische Neigungen üblicherweise nachgesagt werden - 1845 betont: „In der Wirklichkeit der Geschichte ist das menschliche Wesen kein dem einzelnen Individuum innewohnendes $\mathrm{Ab}$ straktum. In seiner Wirklichkeit ist es das ensemble der gesellschaftlichen Verhältnisse. "Die Individualität, die Marx und in seiner Folge die Tradition kritischer Theorie den Abstraktionen des Kapitalismus entgegenhält, ist wesentlich eine gesellschaftliche, die aktiv produziert wird und sich mit den Gesellschaftsformationen wandelt.

Eben deshalb kann im Kapitalismus auch noch nicht gewusst werden, welches die „Fähigkeiten“ und „Bedürfnisse“ (Marx, 1969a:21) sein werden, nach denen eine kommunistische Gesellschaft sich organisiert. Vielmehr ist es kontraproduktiv, sich Gesellschaft und Individualität nach dem Kapitalismus vorzustellen, während man noch ganz in den sozialen und Denkformen des Kapitalismus sozialisiert und verhaftet ist. Deshalb wenden sich Marx und Engels immer wieder gegen Utopien der zukünftigen Gesellschaft, die dem „souveränen Kopf“ (Engels, 1971:248) einzelner Theoretiker*innen entspringen anstatt dem ,geschichtlichen Zusammenhang“, der allein durch Kritik, Negation und (Klassen-)Kampf die „Grundzüge dieser [sozialen] Umgestaltung“ (ebd.) hervorbringt. Utopist*innen hingegen setzten nur, so die Kritik, ihre eigenen Vorstellungen und Interessen absolut und damit die „konservativen oder revolutionären Strömungen [ihrer] Zeit““ (ebd.:91).

In der marxistischen Debatte wird dies als „Bilderverbot“ diskutiert: „Solche Bilderlosigkeit konvergiert mit dem theologischen Bilderverbot. Der Materialismus säkularisiert es, indem er nicht gestattete, die Utopie positiv auszumalen“ (Adorno, 2018:207). Denn, so Adorno (2016:224) anderswo: „Wenn oppositionelle Intellektuelle [...] den Inhalt der Gesellschaft anders wollen, so lähmt sie die Gestalt des eigenen Bewußtseins, die vorweg nach dem Bedarf dieser Gesellschaft modelliert ist.“ Für sie gilt in Bezug auf die Zukunft, was für die Mächtigen heute schon gilt: „Es liegt im Mechanismus der ,pathischen Projektion“, daß die Gewalthaber als Menschen nur ihr eigenes Spiegelbild wahrnehmen, anstatt das Menschliche gerade als das Verschiedene zurückzuspiegeln" (ebd.:118). So wie heute im Namen der Gleichheit die Individualität negiert wird, so würde sie es auch, projizierte man ihren heutigen Begriff in die Zukunft einer anderen Gesellschaft. Deshalb ist Gleichheit kein marxistischer Begriff und wird in der Tradition materialistischer Theorie als abstrakt, verschleiernd und potentiell autoritär kritisiert. Einerseits.

\section{Das emanzipatorische Potential abstrakter Gleichheit}

Andererseits sieht trotz dieser vernichtenden Kritik abstrakter Vorstellungen von Gleichheit bereits Engels (1971:99) auch ihren historischen Sinn und ihre strategische Bedeutung. „Die Gleichheitsforderung im Munde des Proletariats“ ist „,naturwüchsige Reaktion gegen die schreienden sozialen Ungleichheiten, gegen den Kontrast von Reichen und Armen, von Herren und Knechten, von Prassern und Ver- 
hungernden“ und sie kann ,,als Agitationsmittel [dienen], um die Arbeiter mit den eignen Behauptungen der Kapitalisten gegen die Kapitalisten aufzuregen“. Diesen strategischen Einsatz der Gleichheit in politischen Auseinandersetzungen und Kämpfen haben Marx und Engels kaum thematisiert, weshalb er auch in der marxistischen Theorietradition des 20. Jahrhunderts keine große Rolle gespielt hat. Im Zentrum steht er hingegen in jüngeren Debatten um radikale Demokratie, und das aus zwei zusammenhängenden Gründen: Zum einen waren die Erfahrungen mit den bisherigen Versuchen, die kommunistische Gesellschaft real zu entwickeln, desaströs (vgl. Adamczak, 2017), zum anderen ist das von Marx und Engels identifizierte Subjekt ihrer Herbeiführung, das Proletariat, im globalen Norden spätestens seit dem letzten Drittel der 20. Jahrhunderts nicht mehr die treibende Kraft gesellschaftlicher Emanzipation. An seine Stelle trat eine Vielzahl sozialer Bewegungen.

Im Folgenden werden drei prominente Varianten von Theorien radikaler Demokratie kritisch diskutiert, um zu untersuchen, wie sie Gleichheit fassen und dabei über nur abstrakte Gleichheit hinausgehen. Dabei wird gefragt, in welcher Weise die Räumlichkeit von Gleichheit auf der Ebene der Theorie berücksichtigt wird. Zudem werden Aspekte identifiziert, die in eine Konzeption von Gleichheit eingehen sollten, die deren emanzipatorisches Potential beinhaltet.

\subsection{Laclau/Mouffe}

Im Vorwort zur Neuauflage ihres zentralen Werks Hegemony and Socialist Strategy betonen Ernesto Laclau und Chantal Mouffe, dass sie es beim Schreiben als ihre Aufgabe sahen, „dass die Kämpfe gegen Sexismus, Rassismus, sexuelle Diskriminierung und für Umweltschutz mit jenen der Arbeiter*innen in einem neuen linken Projekt artikuliert werden“ (Laclau und Mouffe, 2001:xviii). Ihre Diskurs- und Hegemonietheorie tritt an, um die von ihnen identifizierten Essentialismen und Universalismen der marxistischen Theorietradition $\mathrm{zu}$ überwinden, indem sie sich nicht mit „Gesellschaft“, sondern mit dessen diskursiver Repräsentation, dem „Sozialen“, befassen. Dort fungiert Gleichheit zum einen als die Form, in der radikal-demokratische Inhalte antagonistisch gegen die eingerichtete Hegemonie in Stellung gebracht werden müssen. Politische Bewegungen müssen sich trotz ihrer Unterschiede auf gleiche Forderungen einigen und diese diskursiv um ,leere Signifikanten“ in „Äquivalenzketten“ organisieren. Durch diese Konstruktion eines ,anderen Äquivalenzensystems“ (Laclau und Mouffe, 2020:214) entsteht ein neuer Antagonismus, womit ,die soziale Spaltung auf einer neuen Basis etabliert [wird]“" (ebd.), im Gegensatz zur Hegemonie.

Zum anderen fungiert Gleichheit als Inhalt des antagonistischen Äquivalenzsystems. Mit 1789, der ,,demokratische[n] Revolution“ (ebd.:191), wird „,die Logik der Äquivalenz in das grundlegende Instrument der Produktion des Sozialen transformiert" (ebd.). Seitdem erfolgt in der liberalen Demokratie eine ,äquivalentielle Verschiebung des egalitären Imaginären auf immer umfassendere soziale Verhältnisse“ (ebd.:228). Dass im Namen der Gleichheit Rechte eingefordert und institutionalisiert werden, reicht für eine radikale Demokratie jedoch nicht aus. Denn der Universalismus der Gleichheit vereinheitlicht die notwendig ungleiche „Polyphonie der Stimmen [...], von denen jede ihre eigene irreduzible diskursive Identität konstruiert“ (ebd.:231). Zur Forderung nach Gleichheit muss eine „Reihe von Vorschlägen für die positive Organisation des Sozialen“ (ebd.:228) hinzukommen, die sich zwischen den Stimmen unterscheiden. Radikale Demokratie muss ein je der Situation angepasstes Gleichgewicht ,zwischen einem maximalen Voranbringen der demokratischen Revolution in möglichst vielen Bereichen und der Fähigkeit [...] zur positiven Rekonstruktion dieser Bereiche seitens der untergeordneten Gruppen“ (ebd.:229) anstreben. Beim Radikalisieren der liberalen Demokratie fungiert abstrakte Gleichheit als Ausgangspunkt, von dem aus über sie hinauszugehen ist: „Die Basis einer radikalen Demokratie liegt in der Vervielfältigung der ,öffentlichen Räume“ und ihrer zugehörigen Publika, die über die vom klassischen Liberalismus akzeptierten hinausgeht" (Laclau, 1990:xv, vgl. Mouffe, 2018:27).

Positiv ist von Laclau/Mouffe mitzunehmen, dass sie (als eine der ersten und bis heute am prominentesten) nach Wegen suchen, die Zersplitterung progressiver Gruppen in sozialen Bewegungen zu überwinden, ohne sie zur Aufgabe ihrer Identität zu nötigen. Kritisch ist anzumerken, dass ihre Diskurstheorie tendenziell von realen gesellschaftlichen Verhältnissen absieht (vgl. Farris, 2017:66, die argumentiert, dass die Theorie auf einer ,formalistischen und männlich-zentrierten Dichotomie“ basiert), weshalb die Verbindung zwischen abstrakt formulierter Theorie und politischer Praxis unterbestimmt bleibt und, wichtiger für diesen Beitrag, die Gewordenheit der territorialen Geltung der Gleichheit nicht auf Ebene der Theorie integriert wird. Das Politische scheint nur im vorgefundenen Nationalstaat denkbar. Treffend spricht Sparke (2005:187) von einer ,eingebauten Territorialisierung des Politischen“. Positiv aufzunehmen ist gleichwohl der klare Fokus auf soziale Bewegungen und die Herausforderung, deren kollektive emanzipatorische Kämpfe zusammenzubringen, ohne sie zu vereinheitlichen.

\subsection{Rancière}

Auch für Jacques Rancière (2016a:86) zielt ,,[d]ie demokratische Bewegung [...] darauf ab, die Gleichheit des öffentlichen Menschen auf andere Bereiche des gemeinschaftlichen Lebens auszuweiten“. Und auch für ihn ist das nur ein Teil dessen, was radikaldemokratische Politik ausmacht. Denn die Vorstellung, dass „Ungleichheit eine Realität“ ist und „Gleichheit ein Ziel, das von diesem Ausgangspunkt aus erreicht werden soll“", resultiert in ,einer 
unendlichen Wiederbehauptung der Ungleichheit" (Rancière, 2016b:137). Wahre Politik hingegen „existiert dort, wo die Rechnung/Zählung der Anteile und Teile der Gesellschaft von der Einschreibung eines Anteils der Anteillosen gestört wird. Sie beginnt, wenn die Gleichheit zwischen Beliebigen in die Freiheit des Volkes eingeschrieben wird" (Rancière, 2018a:132). Die hierarchische Ordnung der Gesellschaft wird gestört, sie wird politisiert, wenn jene, deren Stimme nichts zählt, eben diese erheben. Dies tun sie, ob sie es wissen oder nicht, im Namen der Gleichheit, indem sie performativ sich dieselbe Stimme geben, wie sie bislang den Herrschenden vorenthalten war. Gleichheit wird damit nicht durch den Staat zuerkannt, sondern von Menschen selbst eingefordert.

Diese Gleichheit ist zugleich Voraussetzung ihrer Einforderung. Nur durch die „Annahme der Gleichheit“ könne es zum „Bruch mit dem Glauben an die Ungleichheit“ (Rancière, 2016b:139) kommen, der Menschen daran hindert, die Stimme zu ergreifen. Dieser Bruch hat eine Grundlage in der Wirklichkeit der abstrakten Gleichheit:

Sobald der Gehorsam eines Legitimationsprinzips bedarf, sobald es Gesetze geben muss, die sich als Gesetze durchsetzen, und Institutionen, die das Gemeinsame der Gemeinschaft verkörpern, muss jeder Befehl eine Gleichheit voraussetzen zwischen dem, der befiehlt, und dem, der befehligt wird (Rancière, 2016a:73).

Bezugspunkt ist nicht allein die „Gleichheit, wie sie vom Gesetz und seinem Prinzip festgelegt ist" (ebd.:89), sondern, grundlegender, ,[d]ie materielle Idealität der Sprache“ (Rancière, 2018b:51). Die Tatsache, dass sie alle sprechen können, ,weist jede Hierarchie [...] zwischen den Menschen [...] zurück“ (ebd.). Die ,prinzipielle Gleichheit der sprechenden Wesen“ (ebd.:52) äußert sich darin, dass ,in allen Akten des menschlichen Geistes“ (ebd.:27) ,[d]ieselbe Intelligenz [...] am Werk [ist]“ (ebd.). Deshalb gilt: ,Alle Menschen haben die gleiche Intelligenz" (ebd.:29). Diese Gleichheit der Intelligenz lässt sich nicht beweisen. Auf sie verweist aber die Tatsache, dass jene, die kraft ihres Willens ihre Aufmerksamkeit mit Fleiß auf beliebige Lerngegenstände richten, erstaunliche Resultate erzielen. „Der Mensch ist ein Wille, dem eine Intelligenz dient" (ebd.:66). Deshalb tritt die Gleichheit der Intelligenzen erst in der Anwendung des Willens zutage; und dies tut sie wiederum nur, wenn von der o. g. „Annahme der Gleichheit“ (Rancière, 2016b:139) ausgegangen wird.

Erst aufgrund des tätigen Einsatzes des Willens auf Grundlage der Annahme der Gleichheit entsteht eine „Gemeinschaft der Gleichen" (Rancière, 2018b:88), die

die Trennung [...] zwischen denen, die über Intelligenz verfügen, und jenen, die nicht über sie verfügen, ablehnen [würde]. Sie würde nur tätige Geister kennen [...]. Solche Menschen wüssten, dass niemand mit mehr Intelligenz geboren wird als der Nachbar, dass die Überlegenheit, die jemand zeigt, nur die Frucht des Fleißes ist (ebd.).

Die gleiche Intelligenz und die Gemeinschaft der Gleichen stellen sich performativ her, indem sie in Willensakten die eingerichteten gesellschaftlichen Verhältnisse dadurch herausfordern, dass sie ihre Stimme gegen sie erheben - und damit jene Gleichheit realisieren, die sie vorausgesetzt haben und einfordern.

Rancières politische Theorie basiert auf dieser nur performativ sich geltend machenden Gleichheit, die zugleich die Vorstellung abstrakter Gleichheit radikalisiert und dabei über sie auf die Möglichkeit radikaler Demokratie hinausverweist. Anders als bei Laclau/Mouffe erfolgt das nicht mittels diskursiver Verschiebungen, sondern mittels der fortwährenden Einforderung der Gleichheit auf Basis einer anthropologisch anmutenden Annahme gleicher menschlicher Intelligenz. Daran ist insofern anzuschließen, als das aktive Einfordern von Veränderungen durch jene, die keinen Anteil haben, zentral und zugleich festgestellt wird, dass ebendies tatsächlich andauernd stattfindet, sobald sie die Stimme erheben. Allerdings werden damit politische Forderungen tendenziell individualisiert, worauf die Ausführungen zum Verhältnis von Gleichheit, Intelligenz und Wille verweisen. Obschon sich Rancière (2016a:9, 29-34, 86-89) positiv auf Marx' Kritik der Gleichheit bezieht und deren Verkehrung gegen die „Figur des vor Gleichheit trunkenen, demokratischen Verbrauchers" (ebd.:46) scharf kritisiert, verschwinden hinter seinem Fokus auf „Stimme“ und „Intelligenz“ die realen Ungleichheiten „der Geburt, des Standes, der Bildung, der Beschäftigung“ (Marx, 1970:354). Deshalb erteilt er auch Marx und Engels eine Absage, die in der Negation der Widersprüche des Bestehenden den Weg in eine bessere Zukunft wähnen - nicht ohne dabei mit Marx “ Formulierung zur „Wirklichkeit“" des „,menschliche[n] Wesen[s]“ (Marx, 1969b:6) zu spielen: „Die nichtegalitäre Gesellschaft trägt keine egalitäre Gesellschaft in sich. Die egalitäre Gesellschaft ist nichts als das Ensemble egalitärer Beziehungen, die hier und jetzt durch singuläre und prekäre Handlungen geformt werden“" (Rancière, 2016a:142).

\subsection{Balibar}

Die Arbeiten von Étienne Balibar stehen stärker noch als jene von Laclau/Mouffe und Rancière in der Tradition marxistischer Debatten, etwa bezüglich des Form-Begriffs, den er als „Staatsbürgerschaft als politische Form“ (Balibar, 2012:8) und „Nationform“ (Balibar, 1990, 2003) fruchtbar macht. Gleichheit ist für Balibar seit 1789 in Form der "Proposition der Gleichfreiheit“" (Balibar, 2012:8) in die Staatsbürgerschaft als „Spur“ (ebd.:16) eingeschrieben. „Er begreift ,jenen revolutionären " Moment am Anfang der Moderne als entscheidend [...], durch den das ,gleiche Recht ${ }^{*}$ zum Begriff einer neuartigen Universalität wird“ (ebd.:17) 
und in dem die „Einheit (ja sogar Identität der Referenz) der Begriffe von Freiheit und Gleichheit selbst“" (ebd.) enthalten ist. Diese „Gleichung [...] ist nicht essential-

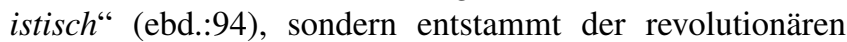
Praxis von 1789. Seitdem zeigt sich immer wieder:

Die Situationen, in denen eine von beiden [Freiheit oder Gleichheit] da ist oder fehlt, sind zwangsläufig dieselben, in denen auch die andere da ist oder fehlt. [...] Die (faktischen) historischen Bedingungen der Freiheit sind genau dieselben wie die (faktischen) historischen Bedingungen der Gleichheit (ebd.).

Die Proposition der Gleichfreiheit ist „,eine irreversible Wahrheit, entdeckt im revolutionären Kampf und durch ihn“ (ebd.), die, einmal in der Welt, „Auswirkungen einer Wahrheit oder Wahrheitseffekte“ (ebd.:94f.) zeitigt. Indem Balibar Gleichheit nicht als Element im diskursiven Kampf um Hegemonie oder als tendenziell individualistische Forderung einer anthropologisch gleichen menschlichen Intelligenz versteht, sondern als in und durch Praxis in kollektiven Kämpfen immer wieder ins Werk gesetzte Wahrheit, bleibt er näher an den realen Kämpfen und Ungleichheiten und damit an der territorialen Verfasstheit des Politischen.

Die Form der Staatsbürgerschaft prozessiert die Widersprüche zwischen Bürger(-rechten) und Mensch(-enrechten). Weil die Bürgerrechte des Bourgeois, der bürgerlich, männlich und Angehöriger der Nation ist, 1789 für alle Menschen beansprucht wurden, werden politische Forderungen nach Freiheit und Gleichheit artikulierbar und die „Gleichung Mensch = Bürger [...] die Bekräftigung eines universellen Rechts auf Politik“ (Balibar, 2012:97):

Zumindest der Form nach [...] eröffnet die Déclaration eine unbegrenzte Sphäre der Politisierung von Rechtsansprüchen, die, jeder auf seine Weise, die Forderung nach Staatsbürgerschaft oder öffentlicher, institutioneller Verankerung von Freiheit und Gleichheit erneuern (ebd.).

Forderungen, die in gesellschaftlichen Kämpfen auf konkrete Inhalte abzielen, werden als solche nach Gleichheit artikulierbar. Deren 1789 institutionalisierte Wahrheit „machen Lohnarbeiter, Frauen, Sklaven, später auch Kolonisierte sich [...] zu Nutze, um rechtliche Ansprüche anzumelden“ (ebd.; vgl.: „Der berühmte Grundsatz, daß alle Menschen gleich seien, läßt in den Kolonien nur eine Anwendung zu: der Kolonisierte wird behaupten, daß er dem Kolonialherrn gleich sei.“ Fanon, 1981 [1961]:37). So treibt die Gleichfreiheit in der Praxis der Kämpfe stets über die Bürgerrechte auf die universellen Menschenrechte hinaus. Dies resultiert in einer ,Dialektik von Aufstand und Verfassung“ (ebd.:20), in der in Revolten neue Rechte im Namen der Gleichfreiheit erkämpft werden, die dann im Recht auf Dauer gestellt werden. Aufgrund dieser unabgeschlossenen
Dialektik ist die Staatsbürgerschaft ,intrinsisch ,fragil ‘ oder ,verletzlich“" (ebd.:24).

Dass aus dieser Dialektik gleichwohl keine fortwährende Ausweitung der Gleichfreiheit folgt, hat drei in der Form der Staatsbürgerschaft selbst angelegte Gründe. Erstens hängen die Erfolge der Kämpfe ,ganz und gar von ,Kräfteverhältnissen ‘ und ihrer Entwicklung unter den

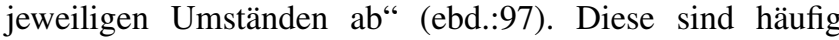
ungünstig, insbesondere seit Beginn der Neoliberalisierungen (ebd.:49-61). Zweitens haben die „organisierten Kämpfe der Arbeiterklasse [...] zur Geburt [einer] ,sozialen Staatsbürgerschaft" beigetragen“ (ebd.:31), die zwar als „Mechanismus universeller Solidarität" (ebd.:36) konzipiert ist, deren Institutionalisierung im Staat aber dazu führt, dass sie ,per definitionem innerhalb nationaler Grenzen konzipiert und umgesetzt wurde“ (ebd.:44). Die Prozessierung des Widerspruchs zwischen Bürger- und Menschenrechten materialisiert sich in einem nationalen Sozialstaat innerhalb territorialer Grenzen.

Damit kommt den Raumformen des Politischen bei Balibar auf Ebene der Theorie zentrale Bedeutung $\mathrm{zu}$, auch wenn er dies nicht raumtheoretisch ausformuliert. Dies ist unter Bezug auf geographische Debatten möglich. Nach Taylor (1994) wurde das Staatsterritorium historisch sukzessive mit politischer Herrschaft, kapitalistischer Reichtumsproduktion, nationaler Kultur und schließlich dem Sozialen i. S. des Wohlfahrtsstaates ,gefüllt“ - und das aus guten Gründen: In dieser Weise stabilisieren der Inhalt (der selbst in sozialen Formen organisiert ist) und die Raumformen sich gegenseitig. Die räumliche Maßstabsebene (Scale) des Nationalstaates erlaubt es, Kompromisse zwischen gesellschaftlichen Antagonismen zu organisieren (Smith, 1984), sein auch durch und gegen Netzwerke konstituiertes (Painter, 2010; Harvey, 2003) Territorium ermöglicht die Organisation von Herrschaft basierend auf dem alleinigen Kriterium der Grenze (Sack, 1983) und die Bedeutung des Staatsterritoriums als „Land, das eine spezielle Bedeutung hat" (Brubaker in: Sturm und Bauch, 2010:186), fungiert in der ,imaginierten Gemeinschaft" (Anderson, 1988) der Nation als ,territoriale Ideologie“ (Murphy, 2005) des Place-Making (vgl. Tuan, 1974). Damit wirken die spezifischen Leistungen der vier Raumformen (vgl. Belina, 2013) des TPSN-Schemas - Territory, Place, Scale und Network -, deren relationale Berücksichtigung Jessop, Brenner und Jones (2008) einfordern, in dem räumlich im nationalen Territorium materialisierten Begriff der „Nationform“ (Balibar, 1990, 2003) zusammen. Sie tun dies in einer Weise, als deren Resultat es als normal erscheint, dass abstrakte Gleichheit als Grundlage der Vergesellschaftung bzw. der sozialen Formen im Nationalstaat nicht für jene gilt, die von jenseits seiner Grenzen kommen.

Denn die Nationform basiert auf „Ausgrenzung“ oder doch zumindest ,ungleiche[m] Zugang zu bestimmten Gütern und Rechten [...], je nachdem, ob man der betreffenden Nationalität angehört oder ,Ausländer" ist" (ebd.:50). 
Allerdings basiert die scheinbar simple Sortierung nach Inund Ausländern bei näherer Betrachtung auf einer konstitutiven Spannung. Denn die demokratische Entscheidung darüber, wer zum Demos gehört, kann demokratisch nur vom Demos selbst gefällt werden. Deshalb gilt: „Der Volkssouverän und der demokratische Staat lassen sich nur zirkulär begründen“ (Demirović, 2009:194). Praktisch geklärt wird die Frage deshalb in einem ,,anstrengende[n] Prozess, bei dem Zugehörigkeit immer wieder neu ausgehandelt und erstritten wird“ (Müller, 2016:22f.). In diesem Prozess spielen räumliche Grenzen eine zentrale Rolle. Deshalb sind sie „die absolut undemokratische[n] oder ,willkürliche[n] ' Bedingungen der demokratischen Institutionen“ (ebd.:156). Weil ihre „Abschaffung [...] zu nichts anderem als einem ,Krieg aller gegen alle' führen dürfte, weil dann alle der ungezügelten Konkurrenz ökonomischer Kräfte ausgeliefert wären“ (ebd.:166), fordert Balibar lediglich eine „Demokratisierung der Grenzen“ (ebd.:187): „für ein Recht auf Grenzübertritt und gegen willkürliche Kontrollen, für die mehrfache Staatsangehörigkeit und gegen die exklusiv-monolithische Staatsangehörigkeit, für die allgemeine Sicherheit und gegen die institutionelle Unsicherheit und Verunsicherung der und vermittels der ,gefährlichen Bevölkerungsgruppen““”(ebd.).

Diese Aussicht ist angesichts der fundamentalen Kritik der Nationform, mit der Balibar (1990) angetreten ist, seltsam unbefriedigend. Einen - zumindest in der Theorie - weiterreichenden Weg weist die Auseinandersetzung mit der dritten Grenze der Dialektik von Aufstand und Verfassung:

Balibar (2012:119) identifiziert zwei ,anthropologische Differenzen", die nicht in der universellen Gleichfreiheit aufgehen: die natürliche „Geschlechterteilung“ (ebd.:108) und die „Trennung von Körper und Geist“ (108), verstanden als jene zwischen geistiger und Handarbeit (Marx und Engels, 1969). Auch wenn das Anthropologische der ersten Differenz unter Bezug auf Butler (1997) in Frage zu stellen ist, sind Balibars Folgerungen aus ihr zentral. Von Ungleichheiten Betroffene, die nicht in der Proposition der Gleichfreiheit aufgehen, bei denen also die Ausdehnung der formalen Gleichheit die Ungleichheit nicht verringert oder sogar vergrößert, müssen ,ihre Befreiung als ,Recht auf Differenz in der Gleichheit" suchen" (Balibar, 2012:110). Hierauf wird im Fazit zurückgekommen.

\section{Jenseits der abstrakten Gleichheit: Gleichheit als Differenz in Solidarität}

Abstrakte Gleichheit ist zentral für die sozialen Formen, in denen das Zusammenleben im Kapitalismus organisiert ist und durch die dessen Widersprüche verschleiert und prozessierbar gemacht werden. Diese Leistung stößt an eine Grenze an den Raumformen, in denen die sozialen Formen organisiert sind. In der Nationform gelten jene jenseits der
Grenze nicht als abstrakt gleich, sondern als fundamental ungleich.

Innerhalb der Nationform wird Gleichheit im Anschluss an die sozialen Formen immer wieder produktiv in emanzipatorischen sozialen Kämpfen eingesetzt, um gleiche Rechte und darüberhinausgehende konkrete Inhalte materieller und anderer Art einzufordern. Dies zeigen die Erfolge von Arbeiter*innen-, feministischen und queeren Bewegungen ebenso wie jene der kolonialen Befreiung. Letztere erfolgte nicht zufällig in Form neuer Nationalstaaten, auch wenn Fanon (1981:208) als antikolonialer Kämpfer forderte, dass ,[n]ur das Nationalbewußtsein, das kein Nationalismus ist, [...] uns eine internationale Dimension zu geben [vermag] " und auch wenn ,die Beziehung zwischen antikolonialen und metropolitanen Nationalismen von gegenseitigen Anleihen, aber auch von erheblichen Differenzen geprägt war" (Castro Varela und Dhawan, 2015:47).

Jenseits der Nationform ist es aufgrund der Raumformen, in denen die sozialen Formen organisiert sind, weit schwieriger, Kämpfe unter Bezug auf Gleichheit zu führen. Dass es möglich ist, zeigen Bewegungen der Sans-Papiers in Frankreich, auf die Balibar häufig Bezug nimmt, oder der Geflüchteten im langen Sommer der Migration 2015.

Die Kritik der abstrakten Gleichheit setzt sie ins Verhältnis zur Art und Weise, in der sich ,[[d]ie Struktur des Wirtschaftssystems [...] auf alle menschlichen Beziehungen und selbst die innerste Verfaßtheit des Individuums aus[wirkt]“, von der Adorno (2019:45) im Eingangszitat spricht. Und sie verdeutlicht, warum die Ungleichheit zwischen „Inländern“ und „Ausländern“, auf die die AfD-Propaganda ebenso setzt wie deren Nachahmer*innen in der selbsternannten politischen Mitte, so plausibel und anschlussfähig ist, ohne damit grundsätzlich das ,universelle Gleichheitspostulat der Aufklärung [...] infrage [zu stellen]“" (Salzborn, 2017:166). Zudem ist die hier vorgeschlagene geographische Kritik der Gleichheit eine, die die wesentliche Räumlichkeit der sozialen Formen des Kapitalismus systematisch auf Ebene der Theorie zu berücksichtigen vorschlägt.

Emanzipatorische Politik muss über die abstrakte Gleichheit nicht nur hinausgehen, sondern artikuliert werden mit dem von Balibar (2012:110) angesprochenen „,Recht auf Differenz in der Gleichheit“". Auch für Adorno (2016:116) wäre ,[e]ine emanzipierte Gesellschaft [...] die Verwirklichung des Allgemeinen in der Versöhnung der Differenzen" und eine „Politik, der es darum im Ernst noch ginge [...] sollte statt auf die schlechte Gleichheit heute [...] den besseren Zustand aber denken als den, in dem man ohne Angst verschieden sein kann“. Diese Übertretung des „Bilderverbots“ scheint ihm notwendig, um gegen die Verabsolutierung der kapitalistischen Abstraktionen die Individualität zu stärken, in der sich, obschon das Individuum selbst ,eine bloße Abstraktion“ (ebd.:170) ist, doch ,ein Stück gesellschaftliches Subjekt“ (ebd.:171) findet, weshalb ,in seiner bündigen Negation, der Abschaffung der Monade durch Solidarität, 
zugleich die Rettung des Einzelwesens angelegt [wäre], das gerade in seiner Beziehung aufs Allgemeine erst ein Besonderes würde" (ebd.:153).

An diese vorsichtige, tastende Annäherung an den utopischen Kern der Art und Weise, wie über bloß abstrakte Gleichheit hinauszugehen wäre, schließt Adamczak (2017:75) in queer-feministischer Erweiterung an, wenn sie an die Stelle der „Beziehungsweise des [kapitalistischen] Wertes, die Allgemeinheit durch Vereinzelung herstellt", jene der Solidarität setzt. In Abgrenzung zur abstrakten Gleichheit fordert sie eine „Gleichheit, um die Grundlage für Solidarität zu gewährleisten“ (ebd.:103), wobei im anzustrebenden ,Ensemble von Beziehungsweisen [...] das Utopische [...] die utopische Gesellschaft ebensowenig zum Maßstab der Subjekte macht wie andersrum die gegenwärtigen Subjekte zur absoluten Begrenzung der utopischen Gesellschaft" (ebd.:54). Auf dem Weg dorthin sind die Überlegungen von Laclau/Mouffe zur diskursiven Zusammenführung progressiver sozialer Bewegungen in ihrer Differenz, sowie jene von Rancière zur Performativität des aktiven Einforderns von Veränderungen durch jene, die keinen Anteil haben, wichtige Aspekte. Mindestens ebenso wichtig aber, so das geographische Argument dieses Beitrags, ist die Infragestellung der Selbstverständlichkeit, mit der Gesellschaft und Rechte anhand der räumlichen Grenzen der territorialen Staaten aufgeteilt werden, um die mittels der Grenzen erst geschaffene Differenz zwischen Nationen absolut $\mathrm{zu}$ setzen und damit die zahlreichen anderen, gesellschaftlich produzierten Differenzen zu negieren. Denn erst diese Verdinglichung der Grenzen macht es möglich, dass das durch Neoliberalisierungen produzierte ,soziale Angstgefühl“" (Hall et al., 1978) sich in einer immer tieferen Spaltung entlang vermeintlich fundamentaler Differenzen entlang räumlicher Grenzen Bahn bricht. An dessen Stelle wären Solidarität in der Differenz anzustreben.

Data availlability. Für diesen Artikel wurden keine Datensätze genutzt.

Competing interests. Der Autor erklärt, dass kein Interessenkonflikt besteht.

Acknowledgements. Für hilfreiche Kommentare zu früheren Versionen danke ich den Herausgebern des Themenheftes „Geographien sozialer Krisen/Krisen sozialer Geographien“ Jonathan Everts und Simon Runkel, Benedikt Korf als Handling Editor, zwei anonymen Gutachter*innen sowie Eva Isselstein, Tino Petzold und Sebastian Schipper.

Review statement. This paper was edited by Benedikt Korf and reviewed by two anonymous referees.

\section{References}

Adamczak, B.: Beziehungsweise Revolution, Suhrkamp, Berlin, 2017.

Adorno, T.: Einleitung in die Soziologie, Suhrkamp, Frankfurt am Main, 2003.

Adorno, T.: Minima Moralia, Suhrkamp, Berlin, 2016.

Adorno, T.: Negative Dialektik, in: Gesammelte Schriften, Band 6, Suhrkamp, Berlin, 7-412, 2018.

Adorno, T.: Bemerkungen zu The Authoritarian Personality von Adorno, Frenkel-Brunswik, Levinson, Sanford, in: Bemerkungen zu „The Authoritarian Personality“ und weitere Texte, Herausgeber: Ziege, E., Suhrkamp, Berlin, 21-70, 2019.

Adorno, T., Frenkel-Brunswik, E., Levinson, D., und Sanford, R.: Einleitung, in: Studien zum autoritären Charakter, Suhrkamp, Berlin, 1-36, 2017.

Anderson, B.: Die Erfindung der Nation, Campus, Frankfurt am Main, New York, 1988.

Balibar, É.: Die Nation-Form, in: Rasse - Klasse - Nation, Herausgeber: Balibar, E. und Wallerstein, I., Argument, Hamburg, Berlin, 107-130, 1990.

Balibar, É.: Sind wir Bürger Europas?, Hamburger Edition, Hamburg, 2003.

Balibar, É.: Gleichfreiheit, Suhrkamp, Berlin, 2012.

Belina, B.: Raum, Westfälisches Dampfboot, Münster, Deutschland, 2013.

Belina, B.: Form analysis, space and spatial struggle, in: Confronting Capital in the 21st Century: Lessons from Marx's Capital, edited by: Silver, M., Palgrave, Basingstoke, 69-89, 2019.

Belina, B.: Social forms, spatial forms, and the New Right, Polit. Geogr., 81, 102091, https://doi.org/10.1016/j.polgeo.2019.102091, 2020a.

Belina, B.: Context and theory, Polit. Geogr., 82, 102229 , https://doi.org/10.1016/j.polgeo.2020.102229, 2020 b.

Blanke, B., Jürgens, U., und Kastendiek, H.: Zur neueren marxistischen Diskussion über die Analyse von Form und Funktion des bürgerlichen Staates, Prokla, H. 14/15, 51-102, 1974.

Brown, W.: Die schleichende Revolution, Suhrkamp, Berlin, 2018.

Butler, J.: Körper von Gewicht, Suhrkamp, Frankfurt am Main, 1997.

Castro Varela, M. und Dhawan, N.: Postkoloniale Theorie: Eine kritische Einführung, transcript, Bielefeld, 2015.

Demirović, A.: Rätedemokratie oder das Ende der Politik, Prokla, 39, 181-206, 2009.

Dietl, S.: Die AfD und die soziale Frage, Unrast, Münster, 2017.

Engels, F.: Herrn Eugen Dühring's Umwälzung der Wissenschaft, in: Marx-Engels-Werke, Band 20, Dietz, Berlin, 1-303, 1971.

Fanon, F.: Die Verdammten dieser Erde, Suhrkamp, Frankfurt am Main, 1981.

Farris, S.: In the Name of Women's Rights. The Rise of Femonationalism, Duke University Press, Durham, London, 2017.

Georgi, F.: Kämpfe der Migration im Kontext, in: Staatsprojekt Europa, Herausgeber: Wissel, J. und Keil, D., Nomos, BadenBaden, 205-227, 2019.

Hall, S., Critcher, C., Jefferson, T., Clarke, J., and Roberts, B.: Policing the Crisis, Macmillan, London, Basingstoke, 1978.

Harvey, D.: The Limits to Capital, Verso, Oxford, UK, 1999.

Harvey, D.: The New Imperialism, Oxford University Press, Oxford, UK, 2003. 
Hirsch, J.: Politische Form, politische Institutionen und Staat, in: Politik, Institutionen und Staat, Herausgeber: Esser, J., Görg, C., und Hirsch, J., VSA, Hamburg, 157-212, 1994.

Intelmann, D.: Sieben Thesen zur urbanen Krise von Chemnitz. Bemerkungen zu den Ereignissen seit dem 26. August 2018, sub \urban, 7, 189-202, 2019.

Jessop, B., Brenner, N., and Jones, M.: Theorizing sociospatial relations, Environ. Plan. D, 26, 389-401, https://doi.org/10.1068/d9107, 2008.

Laclau, E.: New Reflections on The Revolution of Our Time, Verso, London, New York, 1990.

Laclau, E. and Mouffe, C.: Hegemony and socialist strategy, Verso, London, 2001.

Laclau, E. und Mouffe, C.: Hegemonie und radikale Demokratie, Passagen, Wien, 2020.

Lefebvre, G.: 1789. Das Jahr der Revolution, dtv, München, 1989.

Lukes, S.: Egalitarismus, in: Historisch-Kritisches Wörterbuch des Marxismus, Band 3, Argument, Hamburg, 22-31, 1997.

Mainfroy, C.: Gleichheit, in: Historisch-Kritisches Wörterbuch des Marxismus, Band 5, Argument, Hamburg, 838-846, 2001.

Marx, K.: Kritik des Gothaer Programms, in: Marx-Engels-Werke, Band 19, Dietz, Berlin, 13-32, 1969a.

Marx, K.: Thesen über Feuerbach, in: Marx-Engels-Werke, Band 3, Dietz, Berlin, 5-7, 1969b.

Marx, K.: Zur Judenfrage, in: Marx-Engels-Werke, Band 1, Dietz, Berlin, 347-377, 1970.

Marx, K.: Grundrisse der Kritik der politischen Ökonomie, in: Marx-Engels-Werke, Band 42, Dietz, Berlin, 1983.

Marx, K. und Engels, F.: Die deutsche Ideologie, in: Marx-EngelsWerke, Band 3, Dietz, Berlin, 5-530, 1969.

Mishra, P.: Das Zeitalter des Zorns, S. Fischer, Frankfurt am Main, 2017.

Mouffe, C.: For a Left Populism, Verso, London, 2018.

Müller, J.: Was ist Populismus?, Suhrkamp, Berlin, 2016.
Murphy, A.: Territorial Ideology and Interstate Conflict, in: The Geography of War and Peace, edited by: Flint, C., Oxford University Press, Oxford, 280-296, 2005.

Noiriel, G.: Die Tyrannei des Nationalen, zu Klampen, Lüneburg, 1994.

Painter, J.: Rethinking Territory, Antipode, 42, 1090-1118, 2010.

Paschukanis, E.: Allgemeine Rechtslehre und Marxismus, VLP, Berlin, 1929.

Rancière, J.: Der Hass der Demokratie, August, Berlin, 2016a.

Rancière, J.: Method of Equality, in: Recognition or Disagreement, edited by: Genel, K. und Deranty, J., Columbia University Press, New York, 133-155, 2016b.

Rancière, J.: Das Unvernehmen, Suhrkamp, Berlin, 2018a.

Rancière, J.: Der unwissende Lehrmeister, Passagen, Wien, 2018b.

Sack, R.: Human Territoriality: A Theory, Ann. Assoc. Am. Geogr. 73, 55-74, 1983.

Salzborn, S.: Angriff der Antidemokraten, Juventa, Weinheim, Basel, 2017.

Smith, N.: Uneven Development, Blackwell, Oxford, 1984.

Sparke, M.: In the Space of Theory, University of Minnesota Press, Minneapolis, USA, 2005.

SPON: Seehofer laut Medienberichten „Mutter aller Probleme ist die Migration“, online aufrufbar: https://www.spiegel.de/politik/deutschland/horst-seehoferlaut-medienberichten-mutter-aller-probleme-ist (letzter Zugriff: 17 September 2020), 2018.

Sturm, T. and Bauch, N.: Nationalism and Geography: An Interview with Rogers Brubaker, Geopolitics, 15, 185-196, 2010.

Taylor, P.: The state as container: territoriality in the modern worldsystem, Prog. Human Geogr., 18, 151-162, 1994.

Tuan, Y.: Space and Place: Humanistic Perspective, Prog. Geogr., 6, 211-252, 1974.

Zick, A., Küpper, B., und Berghan, W.: Verlorene Mitte Feindselige Zustände. Rechtsextreme Einstellungen in Deutschland 2018/19, Dietz, Bonn, 53-116, 2019. 